\title{
Gradhiva
}

GRADHI

Revue d'anthropologie et d'histoire des arts

$7 \mid 2008$

Le possédé spectaculaire

\section{Yves Laloy, un dialogue entre primitivisme et surréalisme}

Yves Laloy. Primitivism and surrealism: a dialogue between two cultures

Suzanne Duco

\section{(2) OpenEdition}

Journals

Édition électronique

URL : http://journals.openedition.org/gradhiva/1115

DOI : 10.4000/gradhiva. 1115

ISSN : 1760-849X

Éditeur

Musée du quai Branly Jacques Chirac

Édition imprimée

Date de publication : 15 mai 2008

Pagination : 134-143

ISBN : 978-2-915133-86-8

ISSN : 0764-8928

Référence électronique

Suzanne Duco, «Yves Laloy, un dialogue entre primitivisme et surréalisme », Gradhiva [En ligne],

7 | 2008, mis en ligne le 15 mai 2011, consulté le 03 mai 2019. URL : http://journals.openedition.org/ gradhiva/1115; DOI : 10.4000/gradhiva.1115

(c) musée du quai Branly 


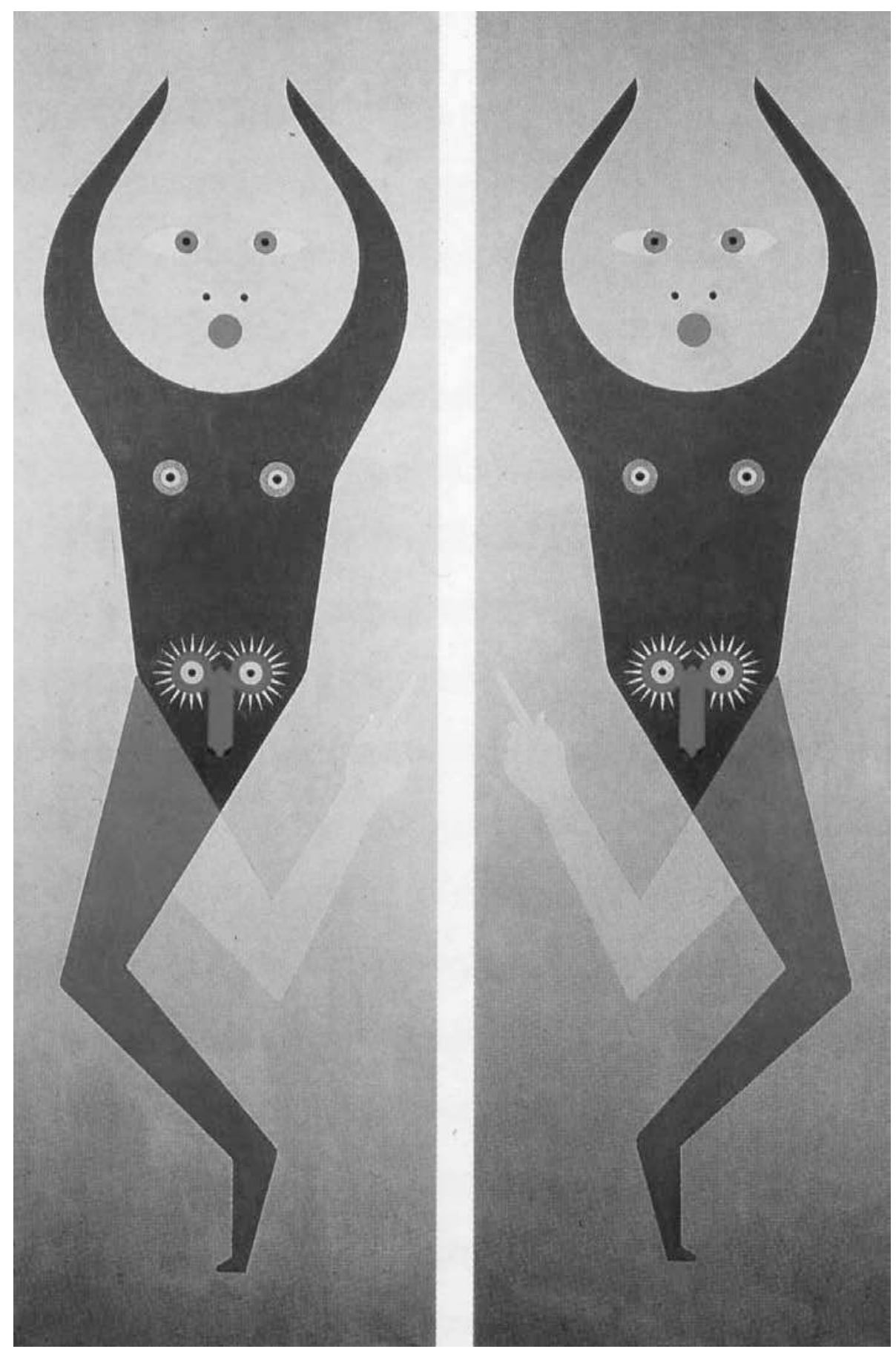

Fig. 1 Yves Laloy, sans titre, dyptique (les danseurs), huile sur toile, 1965. Collection Carl Laszlo, Bâle. 


\section{Yves Laloy}

\section{Un dialogue entre primitivisme et surréalisme}

Suzanne Duco*

$\mathrm{A}$ ndré Breton a été l'un des premiers à attirer l'attention du public sur l'œuvre d'Yves Laloy (1920-1999) à la fin des années 1950. De nombreuses facettes de son œuvre ont en effet un lien étroit avec le surréalisme : des formes géométriques ou organiques explosives, des visages ou des personnages déstructurés, grotesques ou tragiques, enfin des tableaux fondés sur des jeux sémantiques et phonétiques. Les constructions géométriques relèvent plus d'un «abstractivisme rythmique et lyrique ", selon l'expression d'André Breton (Breton et Legrand 1991 [1957] : 148), que d'une épure d'architecte. Quant aux peintures figuratives, elles contiennent des thèmes surréalistes, comme l'œil et le double. L'association de Laloy au surréalisme tardif, d'une certaine façon certifiée par Breton luimême choisissant le tableau Les petits pois sont verts... les petits poissons rouges afin d'illustrer la couverture de l'ouvrage Le Surréalisme et la Peinture, ne doit pas conduire à minimiser une importante source d'inspiration de cette œuvre : les arts non occidentaux, et ceux des Amériques en particulier. Breton avait parfaitement conscience de ce rapprochement lorsqu'il écrivait à propos du peintre : " L'arc-en-ciel à tête humaine qui cerne les admirables tableaux de sable des Indiens navajo de l'Arizona semble présider à la création de l'œuvre d'Yves Laloy. » Et plus loin : " Alors qu'une composition de Kandinsky répond à des ambitions symphoniques, un tableau de sable navajo relève avant tout de préoccupations cosmogoniques et tend à influencer, de manière propitiatoire, le cours de l'univers. Le propre de l'œuvre d'Yves Laloy est de ne faire qu'une de ces deux démarches si distinctes " (Breton 1965 : 254). C'est précisément cette idée que nous tenterons d'illustrer ici. Laloy est préoccupé d'un côté par des questions de composition formelle (" symphoniques » si l'on adopte la métaphore de Breton), mais il est aussi soucieux de donner à son art une dimension magique ou religieuse, en tout cas spirituelle (ou " cosmogonique » si l'on suit Breton). Laloy était en effet un esprit religieux, empreint de catholicisme. Paradoxalement, cette quête religieuse permet de mieux comprendre la relation de ce peintre au surréalisme et aux arts non occidentaux, qui sont pourtant bien étrangers au christianisme : il y cherche le sacré. Cette thématique apparaît particulièrement bien dans deux types de peintures de Laloy que nous aborderons successivement, à savoir d'abord les œuvres géométriques puis les œuvres anthropomorphes.

* Suzanne Duco prépare actuellement le catalogue raisonné des œuvres d'Yves Laloy. 


\section{Géométrie rythmique et magie}

Dans les œuvres géométriques d’Yves Laloy, le dessin est complexe, fait de lignes brisées, de zigzags. Par une composition dynamique et une gamme colorée très riche, l'artiste crée une géométrie rythmique (II et III). La répétition des motifs sans symétrie et la stridence des couleurs impriment au tableau mouvement et violence.

Ses œuvres évoquaient à André Breton les tableaux de sable des Indiens navajo, on l'a vu. Le poète avait été vraisemblablement subjugué par un espace très dynamique, par l'imbrication des formes, l'extrême densité du dessin, mais aussi par le choix des couleurs et le choc des tonalités. À propos de cette composition et de quelques autres similaires, il parlait de "scintillement stellaire ", faisant allusion non seulement à l'effet optique induit par la construction, mais aussi à l'image d'un croissant de lune flottant dans l'espace de la toile. L'ensemble a dû exercer sur le poète un enchantement quasi magique, au sens que Freud donnait à la magie, à savoir celui d'une domination des hommes et des choses par «la toute-puissance des idées ». Breton et Gérard Legrand, dans L'Art magique, explorent l'histoire de l'art depuis les périodes primitives jusqu'à Paul Gauguin, en passant par les visions hallucinantes d'un Jérôme Bosch ; ils trouvent là les racines du surréalisme. La magie est pour ces auteurs inspirés par Novalis, à qui ils se réfèrent, «l'ensemble des opérations humaines ayant pour but la domination impérieuse des forces de la nature par le recours à des pratiques secrètes de caractère plus ou moins irrationnel " (1991 [1957] : 106). L'art abstrait, tout comme l'art figuratif, peut, selon Breton, exercer sur l'homme du xxe siècle une sorte d'envoûtement proche de la magie.

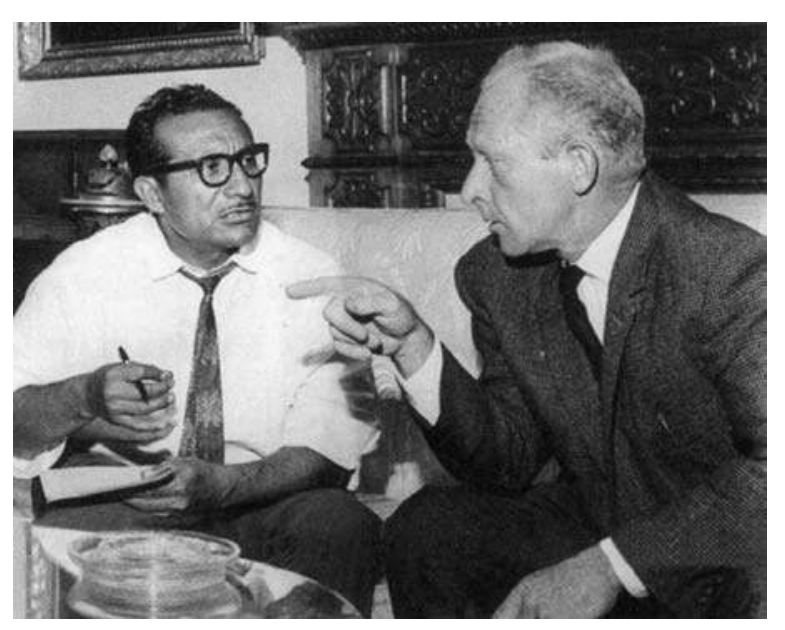

Fig. 2 Yves Laloy (à droite) lors de son entretien avec un journaliste de Lima, janvier 1965.
Aussi n'est-il pas étonnant que ce dernier choisisse Les petits pois sont verts... les petits poissons rouges pour orner la jaquette de son livre Le Surréalisme et la Peinture. Cette représentation insolite réunit tous les éléments déclencheurs de la magie : analogie irrationnelle mise en image par la confusion du vivant et de l'inerte, métaphore picturale et verbale, jeu phonétique et sémantique. L'irrationnel engendre la magie et, selon Breton, l'image analogique est l'un des moyens pour y parvenir (1968 [1947] : 10). L'hommage du poète à Laloy, qui paraît dans Le Surréalisme et la Peinture (Breton 1965 : 254-256), entre en résonance avec la première phrase du livre, devenue célèbre : « L'œil existe à l'état sauvage [...] » (ibid. :1-2). Avec les mêmes mots, les mêmes images, Breton dit, dans cet éloge du regard et de la vision, son admiration pour Laloy et les peintres qui savent regarder, qui « voient » au-delà du visible ${ }^{1}$. C'est cette « clairvoyance hors de pair " qui conduirait Laloy à créer une figuration déconcertante ou une abstraction des plus débridées.

Irrationnelles dans l'agencement des formes, d'un chromatisme souvent agressif, les compositions abstraites de cet artiste pourraient être dictées par l'inconscient. Laloy disait d'ailleurs peu de temps avant sa mort: "[...] quand je vois mes toiles, je me demande ce que j'avais dans la tête quand j'ai fait ça [...]. Ces choses sont du domaine de l'inconscient. " Cet artiste a construit un univers énigmatique " prenant le jour non plus sur le monde extérieur, mais intérieur » (ibid. : 256). Cette intériorité, présente au sein même d'un monde de signes, se manifeste par des constructions rebelles à toute logique géométrique ou décorative. L'œuvre de Laloy relève d'une démarche qui s'apparente aux préoccupations cosmogoniques des Indiens navajo. Si, au plan formel, les similitudes sont évidentes, ce rapprochement est avant tout d'ordre symbolique (I). Leurs peintures sèches, réalisées uniquement à des fins cérémonielles, sont indissociables des rituels invoquant les dieux et les ancêtres. Elles traduisent la vision d'un cosmos où tous les êtres sont dépendants les uns des autres. Exécutées au cours d'une seule journée dans un but précis, la guérison d'un malade ou le rétablissement de l'harmonie spirituelle d'un patient, elles étaient détruites après usage. Nous ne les connaissons que par des observateurs blancs qui en ont fait des copies sur carton. Leur composition est très éla-

1. L'étude du seul vocabulaire montre l'importance qu'André Breton accorde au regard et à la qualité de la vision. Le mot «œil » revient trois fois sous sa plume dès la première page consacrée à Yves Laloy. L'image de l'arc-en-ciel est présente dans les deux textes (1965 et 1968) et les «fenêtres lumineuses » renvoient à la métaphore du tableau-fenêtre. 
borée. Ces œuvres comportent des personnages ou des êtres mythiques géométrisés, des représentations de nuages ou d'éclairs, des animaux stylisés, des motifs géométriques d'une grande finesse. L'ensemble tend vers la symétrie ou, tout au moins, montre une répartition équilibrée des motifs. Comme chez Laloy, un sens esthétique proche de l'ornementation se double d'une démarche spirituelle. On y retrouve une même précision du dessin, une géométrisation semblable des formes ainsi qu'une harmonie des couleurs similaire. Sur un fond sableux doré, les Navajo utilisaient les couleurs naturelles provenant de pétales de fleurs, de farines de céréales ou de charbon de bois. Les teintes ocre, rouges et noires choisies par Laloy s'en rapprochent. Au plan formel, une différence cependant : la dissymétrie chez Laloy, une symétrie presque parfaite chez les Indiens navajo. Bien après Breton, William Rubin, en 1984, à l'occasion de la grande exposition du MoMa, Primitivism in the zoth century art, souligne les affinités profondes qui unissent une grande partie de l'art contemporain à l'art tribal, précisant qu'au-delà des similitudes formelles, les objets primitifs peuvent exercer un pouvoir magique (catalogue Rubin 1984 : 36). Deux ans plus tard, l'exposition de Los Angeles The Spiritual in Art, abstract painting 18901985 (catalogue Tuchman 1986) met l'accent sur une nouvelle manière d'aborder l'abstraction et rappelle que la dimension spirituelle inhérente à l'art des Indiens d'Amérique a été remarquée par des artistes tels que Jackson Pollock et Wolfgang Paalen.

S'agissant de Laloy, la plupart des œuvres de l'artiste susceptibles d'être confrontées à des objets ou des textiles extra-européens ont été réalisées entre 1953 et 1959, bien avant 1965 - date de son voyage au Pérou. Néanmoins, il s'était documenté sur les pays qu'il n'avait pu visiter. Il intitule Montez-Uma (III) un tableau géométrique peint en 1953. Peut-être avait-il été frappé par le caractère belliqueux des deux empereurs aztèques du même nom? Ou bien avait-il gardé en mémoire des reproductions d'objets de l'art aztèque qu'il avait vues à l'École des beaux-arts? Dans La Beauté et ses à-côtés (fig. 3), Laloy consacre un chapitre à la magie et au surréalisme². Il évoque l'effigie du célèbre dieu Quetzalcóatl du temple des guerriers de Chichen-Itza. Il s'agit, dit-il, « d'un être fantastique conciliant l'homme, l'oiseau et le serpent. Lors de l'arrivée des Espagnols au Guatemala, celui qui tuait un quetzal était puni de mort. Les plumes de cet oiseau passeraient encore pour avoir certaines vertus magiques aux yeux des tribus indiennes. Dans un musée de Vienne, est conservée la coiffure royale en plumes de Quetzal offerte par l'empereur Moctezuma à Cortés en

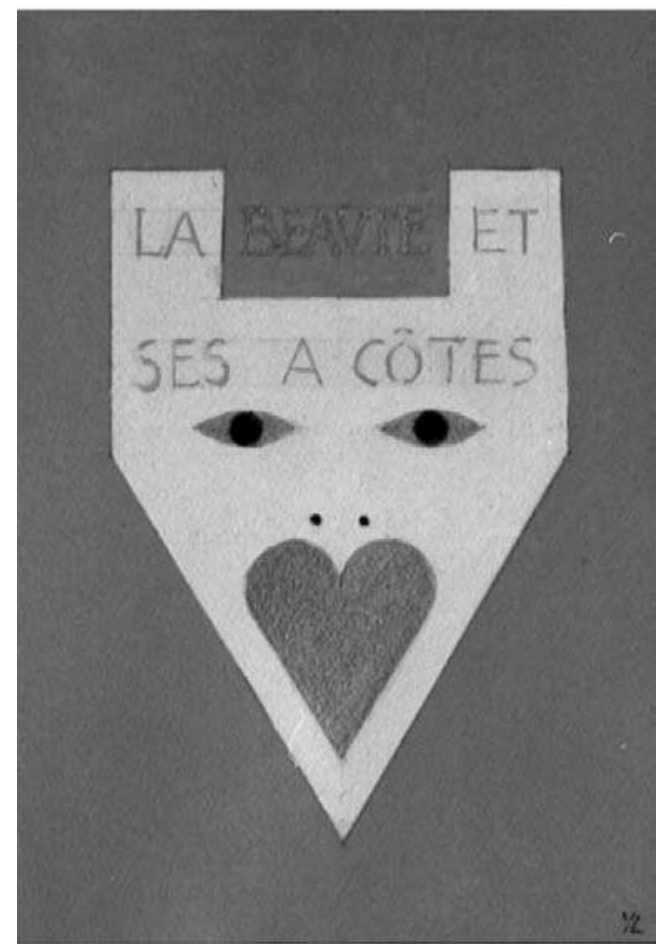

Fig. 3 Yves Laloy, La Beauté et ses à-côtés, 1955. Collection particulière.

1519 ». Sans doute Laloy avait-il une connaissance livresque des arts du Nouveau-Mexique et de l'Arizona. José Pierre3 ${ }^{3}$ qui refusait de voir dans l'œuvre de Laloy des influences, d'où qu'elles viennent, écrivait cependant : « Les traces de pas [...] sont à chercher sur le sol des grottes souterraines où se trouve le cours des rivières perdues » (1967:2). Cette image des réminiscences surgies des tréfonds de la mémoire offre des pistes d'interprétation sur la question des liens existant entre l'art géométrique du peintre et l'art extra-européen en général.

2. Yves Laloy a écrit La Beauté et ses à-côtés durant plusieurs années. Ce livre, comprenant trente chapitres, n'a, à son grand regret, pas été publié. Nous n'avons pu consulter le manuscrit, mais nous en connaissons quelques extraits ainsi que les têtes de chapitre, dont quelquesunes sont révélatrices des préoccupations de l'auteur:

L'œil - Rêve - Vision - Apparence et réalité

Magie - Surréalisme

Action et contemplation

Existence et nature du Beau

Formation et perception de l'image - Actualité de l'image Sa puissance

Le corps chez les Anciens - Le corps aux yeux des Saints

3. José Pierre, écrivain et critique d'art, a fait partie du cercle d'André Breton à partir des années 1950. II a participé aux grandes expositions du surréalisme et a écrit de nombreux ouvrages sur ce mouvement, s'intéressant plus particulièrement aux arts plastiques. II a contribué à faire connaître l'œuvre d'Yves Laloy, dont il appréciait la spontanéité et «la qualité de l'inspiration » (préface de l'exposition Yves Laloy, Milan, 1967). 
D'autres œuvres, au dessin moins complexe, entretiennent des rapports étroits avec des objets d'Amérique. Un tableau peint en 1955, basé sur une succession de triangles, présente ainsi des similitudes dans la forme et même dans la palette chromatique avec les tapas océaniens et les textiles «à effet optique éblouissant " des Indiens navajo. Les lignes en dents de scie et les couleurs vives sont très proches des formes et des couleurs des créations textiles navajos des années $1870-$ 1890 ainsi que des châles du Pérou et des parures mayas. Quelques peintures au dessin plus dépouillé privilégient les verticales. La simplicité des motifs, leur répétition peuvent être mises en parallèle avec des objets d'Amérique du Nord - un parflèche en cuir (v) ressemble ainsi à une toile de Laloy peinte en 1969 (IV). Un tableau tardif (vI) pourrait avoir été inspiré par des poteries incas exposées dans les musées de Cuzco ainsi que par les jarres pueblos du Nouveau-Mexique (vII). Cette peinture, réalisée en 1983, est asymétrique. Elle agresse le regard par la violence de la gamme chromatique et par la dynamique des formes acérées, qui alternent avec des motifs en losange et en damier. Une comparaison formelle met en évidence la similitude avec la poterie pueblo, caractérisée par une géométrie basée sur des zigzags et des espaces en damier. La composition est elle-même héritée des vanneries fabriquées il y a plusieurs siècles par les anciens Pueblos. Bien que Laloy ne se soit jamais exprimé sur la genèse de son art, l'affinité graphique, dans cet exemple, est évidente.

Enfin, s'agissant plus particulièrement des compositions labyrinthiques de Laloy, un rapprochement peut être fait avec l'art des molas créé par les Indiens kuna, vivant pour la plupart au Panamá sur une quarantaine d'îlots coralliens de l'océan Atlantique (catalogue Tableaux kuna 2003 : 99). Cet art, de tradition amérindienne, est décoratif. Les créations textiles, réalisées par des femmes, sont destinées à l'ornement des corsages des vêtements féminins. Les motifs géométriques, les arabesques, les tracés labyrinthiques y sont dominants. À cet aspect ornemental s'ajoute une signification symbolique, le terme mola désignant la "paire" ou le " double ", d'où, très souvent, une symétrie dans le dessin et dans les deux faces du corsage. Aspect décoratif et signification spirituelle ne sont pas antinomiques : les Indiennes kuna réalisaient leurs créations textiles dans le plus grand secret, ne les dévoilant qu'une fois réalisées. Les Kuna associaient l'image du labyrinthe à l'idée que l'homme, la végétation et les animaux sont toujours reliés par des chemins complexes. La croyance en une intrication des espèces et des milieux, à la base des compositions labyrinthiques des Kuna, est aussi une idée chère aux surréalistes. Ainsi, sur le plan strictement formel, les compositions orthogonales ou labyrinthiques de Laloy évoquent certains molas. Dans une lettre à ses parents, non datée, l'artiste écrit :

Ma peinture repose sur un dessin linéaire : ce sont des traits qui composent un labyrinthe parcouru par le fil d'Ariane, qui est le fil de la pensée. Dans ce labyrinthe, je me perds et je me sauve. Parti d'un point, c'est par une suite de points que je reviens au même point : ainsi, ce que je peins, en un sens ne m'avance à rien. J'ai représenté un circuit fermé [...], ce que j'ai figuré, c'est un cycle, c'est une étape.

Pour l'artiste, la géométrie complexe du labyrinthe est un moyen d'exprimer le chemin sinueux de sa pensée, les avancées et les reculs d'une intelligence à la recherche de la vérité. C'est le fil tendu à Thésée par Ariane pour se libérer du Minotaure. Il s'agit pour Laloy d'un itinéraire non clos qui doit déboucher sur un univers autre. À propos des architectures primitives, Breton, dans L'Art magique, soulignait le caractère énigmatique de l'itinéraire tortueux et inachevé du labyrinthe : « La caverne et le labyrinthe remplissent une fonction analogue à la tour et au temple : ils sont également des condensateurs de force magique, mais dirigés vers les effluves telluriques » (Breton et Legrand 1991 [1957] : 135). Convergent ainsi dans ces peintures de Laloy une thématique de la culture classique européenne revisitée par les surréalistes et une structure formelle proche de l'art d'un peuple amérindien. La peinture figurative de Laloy, en particulier certaines représentations anthropomorphes que nous présentons à la suite, offre d'autres exemples d'une telle fusion créative.

Corps dénudés, visages masqués: art amérindien et surréalisme

L'abstraction n'est qu'une facette de l'art d'Yves Laloy, qui utilise aussi les ressources de la figuration. Ainsi, il représente, ou plutôt, suggère, des corps et des visages schématisés, déstructurés, parfois géométrisés et toujours disproportionnés. Il utilise un vocabulaire original, constitué de traits simplifiés à l'extrême et d'aplats de couleurs vives. La frontalité de la représentation donne un aspect énigmatique ou terrifiant à ses personnages et à ses visages. Réalisés le plus souvent autour des années 1955-1960, les tableaux ou les objets peints reprennent d'une manière nouvelle les thèmes universels traités par les surréalistes : l'œil et le regard, l'œil et le sexe, l'obsession du double, l'anthropomorphisme 
et la confusion des espèces, la mutation de l'objet. Laloy tente d'appréhender les êtres en cherchant à y découvrir une vérité autre que celle de leur enveloppe apparente. Il l'a fait avec une iconographie en affinité avec certains aspects de l'art tribal. Les surréalistes - et notamment André Breton, Benjamin Péret, Max Ernst, Kurt Seligmann, Wilfredo Lam, Wolfgang Paalen, etc. se sont intéressés notamment aux arts de l'Amérique et de l'Océanie en ce qu'ils privilégient le monde de l'imaginaire, les mythes ou encore l'activité onirique. Certaines œuvres figuratives de Laloy, à travers les thèmes évoqués plus haut, peuvent être regardées à la fois comme une assimilation du surréalisme et une réappropriation de l'art tribal. Dans les documents rapportés de Bogotá, Laloy fait d'ailleurs quelques commentaires sur l'art colombien, accompagnés de dessins (fig. 4). Des parallèles sont possibles, qui résultent d'un examen des formes utilisées et des sujets explorés par le peintre. Abordons rapidement quelques-uns de ces motifs figuratifs.

L'œil, et plus précisément le regard, a fasciné Laloy. Les visages et les yeux stylisés à l'extrême sont d'une étonnante expressivité. Le regard est intense, scrutateur, et exprime une certaine violence. La transformation du visage en masque tragique, exprimant l'effroi ou la stupéfaction, est aussi l'un des procédés couramment utilisés par Laloy, par exemple dans Marie l'Égyptienne (viII), dont le portrait a quelque chose d'insolite par son expression hiératique. Réalisée après un voyage en Égypte, cette représentation bouscule l'iconographie classique de la Vierge. Le visage noir, au regard sévère, est entouré d'un objet trivial - une houe clouée sur le support en bois du tableau - qui tient lieu d'auréole. Outre son séjour au Caire, le tableau Marie l'Égyptienne a pu être motivé par la fascination de Laloy pour le visage et son contraire, le masque. Bien que la ressemblance ne soit pas frappante, le rapprochement est possible avec les traits outranciers des visages dans tous les masques des Amériques, et notamment avec ceux de Colombie-Britannique. Citons, par exemple, plusieurs masques à transformation kwakiutl, qui évoquent le masque peint par Laloy, ainsi que le masque articulé haida anthropomorphe, qui appartenait à André Breton et qui est depuis peu conservé au musée du quai Branly (VIII). En 1960, Laloy peint un tableau où sont inscrits les mots "Ouah Ouah ». Plusieurs espèces, végétales et animales, s'y côtoient, l'ensemble paraissant issu de visions oniriques. Un visage aux dents apparentes et aux yeux apeurés est entouré de flammes. Cette violence dans le regard fait penser à un grand nombre de masques de
Nouvelle-Guinée ou d'Alaska, comme le masque yup'ik, mi-poisson mi-homme, à la bouche semi-édentée que Breton avait acheté lors de son séjour à New York. Dans un registre plus apaisé, Laloy a peint pour la couverture du livre qu'il souhaitait publier un portrait où il écrit les mots : «La Beauté et ses à-côtés ». Ce portrait a les contours d'un masque triangulaire et la bouche, la forme d'un cœur.

Derrière le masque, il y a l'obsession du double. La dualité de l'être humain marque une grande partie de la production artistique de Laloy. Sous forme de boutade, le peintre écrivait : «L'homme, extérieurement apparaît symétrique, mais intérieurement il ne l'est pas, puisque son cœur est à gauche. L'art, dont le rôle est d'exprimer l'homme, ne sera jamais qu'une illusion d'optique qu'il faut accepter comme telle. » Il s'agit bien d'exprimer l'homme dans sa totalité, mais aussi dans sa complexité. C'est cette part cachée de l'être humain que le peintre doit révéler. Selon Baudelaire, c'est à cette dualité même que l'on reconnaît le véritable artiste : " [...] l'artiste n'est artiste qu'à la condition d'être double et de n'ignorer aucun phénomène de sa double nature " (1980 [1857] : 701). Laloy exprime cette double nature de plusieurs manières : la déstructuration des visages et des corps, l'apparition d'un double visage, enfin la représentation de deux personnages dont l'un est le double de l'autre. La plupart de ses personnages mettent en évidence leurs attributs sexuels. Les corps, déformés ou stylisés, sont mis à nu. Mais cette nudité révèle un double ou un triple visage. Ainsi, lorsqu'il écrit Je suis breton, je suis la loi, vive la France, viva el Perú sur la toile qu'il peint en 1965 (VIII) pour marquer son séjour à Lima, l'artiste inscrit sur le torse en forme de tête de bovidé un visage qui se juxtapose à la véritable tête du personnage, ridiculement petite. Quant aux bras, ils se confondent avec les cornes de l'animal. L'artiste choisit les couleurs chaudes de l'Amérique latine : jaune, ocre et noir. La raideur des formes, la frontalité de la représentation peuvent évoquer certaines figurines péruviennes, et la position des bras est similaire à celle d'un crochet de Nouvelle-Guinée (viII). L'originalité de cette représentation réside dans le choix de ce corps mi-homme, mi-animal, ainsi que dans ce clin d'œil de l'artiste à son arrivée à Lima dans un camion transportant des bovins.

L'obsession du double a hanté cet artiste, obsession présente aussi chez plusieurs artistes surréalistes comme Victor Brauner, E.L.T. Mesens ou Enrico Baj. Deux tableaux peints en 1960 évoquent deux visages géométrisés, présentés frontalement, presque identiques dans leur 
composition et leurs dimensions, séparés par un personnage également géométrisé. Un titre est inscrit au dos du premier tableau : Le Mal est mon seul Bien. Dans le second, Laloy écrit sur la toile : Après tout, la mort n'est que ce qui vient après. Après vous!! Après tout!! L'opposition de deux principes contraires, le mal et le bien, la vie et la mort, fait partie des obsessions de l'artiste, qui pourrait s'être référé au thomiste Jacques Maritain lorsqu'il affirmait : " L'homme est la cause première du mal; il est notre création. " Mais la composition nous force à chercher une autre piste possible. Le caractère hiératique des corps et des visages, la transformation de ces derniers en masques de tragédie, la géométrie triangulaire ainsi que la palette chromatique, en un mot la force d'expression, évoquent des sculptures ou des poteries péruviennes. Quant aux doubles personnages, on les retrouve notamment au Mexique. Otto Rank relate que la véritable divinité dans le jeu de paume mexicain était l'image double du dieu Xoloth, le nom de Xoloth signifiant « celui qui apparait par paire, le jumelé ». Ce dieu est également considéré comme celui qui guide le Soleil et la Lune dans les enfers, « comme le guide de la vie et de la mort » (1998 [1932] : 252). L'art mexicain a souvent représenté dans les sculptures le motif de la double figure, avec deux divinités antinomiques : le dieu du Vent (la vie) et un dieu à tête de squelette (la mort). Si l'on se tourne vers l'art amérindien, on constate que beaucoup de peintures sèches des Indiens navajo sont présentées par paires, conformément à la vision du monde de ces populations pour lesquelles un système équilibré de forces opposées doit être maintenu pour éloigner la souffrance et le mal. Ainsi sont représentés le soleil et la pluie, la lumière et la nuit, le père et la mère, le ciel et la terre. L'un de ces tableaux de sable, Father sky and Mother earth'4, peut être mis en regard avec les deux tableaux de Laloy mentionnés plus haut - les corps géométrisés, leur frontalité, leurs formes symétriques, seulement différenciées par les couleurs; leur symbolique enfin, qui est ici double : le père et la mère, le ciel et la terre. Ces éléments nous permettent de mettre en parallèle des œuvres qui peuvent paraitre tellement opposées : d'un côté le tableau d'un artiste français contemporain, qui fait référence à la foi chrétienne; de l'autre une œuvre qui fait partie intégrante d'un rite indien destiné aux dieux et aux esprits.

Enfin, deux tableaux en forme de diptyque retiendront notre attention : d'une exceptionnelle dimension $(200 \times 65 \mathrm{~cm})$, peints en 1965 après le séjour à Lima, ils ne portent aucun titre (fig. 1). L'iconographie reprend partiellement celle du tableau Je suis breton, je suis la loi, vive la France, viva el Perú. Le haut du corps des personnages se confond avec une tête de bovidé. Les cornes, qui épousent la forme du visage, peuvent aussi être assimilées à des bras. Cependant, l'une des jambes de chaque personnage est en réalité un bras dont la main est dirigée vers le haut; en outre, un troisième visage se superpose aux attributs sexuels masculins. Ces pantins ou ces danseurs peuvent être à plus d'un titre rattachés à une imagerie surréaliste (le regard, le sexe, l'humour, etc.). Ils sont notamment l'expression de la hantise du double, l'un des personnages étant exactement le calque de l'autre. Rapprochons cette peinture, non pas d'une œuvre d' « art primitif », mais d'une chromolithographie exécutée en 1867 par George Catlin, dans laquelle cet ethnologue et peintre représentait deux Danseurs mandan personnifiant le jour et la nuit 5 . Même composition, même attitude, à la fois symétrique et inversée : l'un des danseurs lève la main gauche et le pied gauche, l'autre la main droite et le pied droit; l'un est peint en noir, l'autre en rouge, et nous savons que le premier symbolise la nuit et le second la lumière. Ces Indiens, Catlin les avait vus danser, il les avait peints et ceux-ci faisaient partie, selon sa propre formule, de ses «tableaux vivants". Nous savons que la danse a partie liée avec la magie; les danseurs peints par Catlin, comme les énigmatiques pantins de Laloy, soulèvent des interrogations par leur force suggestive. Une équivalence formelle et intellectuelle est mise en évidence par le regard croisé que l'on peut porter d'une œuvre à l'autre. Même sans influence directe, ces œuvres si éloignées produisent un même effet magique.

Chacun peut s'interroger sur les sources d'inspiration de Laloy, mais c'est avant tout sur les modes d'appropriation et de reformulation d'éléments esthétiques divers qu'il faut s'interroger dans son cas. Un grand artiste n'est pas « inspiré par », « il s'inspire de ». Ce n'est pas un sujet passif, mais un architecte qui construit à partir de matériaux collectés çà et là. L'œuvre singulier, déconcertant, de Laloy illustre bien cette créativité. Un attrait pour la géométrie, une foi religieuse profonde, un questionnement sur le macrocosme et le

4. Father sky and Mother earth, reproduction extraite de Mark Bahti (2003). Cet auteur cite ses sources dans la bibliographie de l'ouvrage : Griffen-Pierre Trudy, Earth is my mother, sky is my Father. Space, time and astronomy in Navajo sand Paintings. Albuquerque, University of New Mexico Press, 1995.

5. Cette œuvre de George Catlin figure dans la revue Gradhiva, $n^{\circ} 3$, nouvelle série, 2006, planche XIII. Elle est reprise en filigrane au début de chaque chapitre de cette revue consacrée au musée indien de George Catlin. 

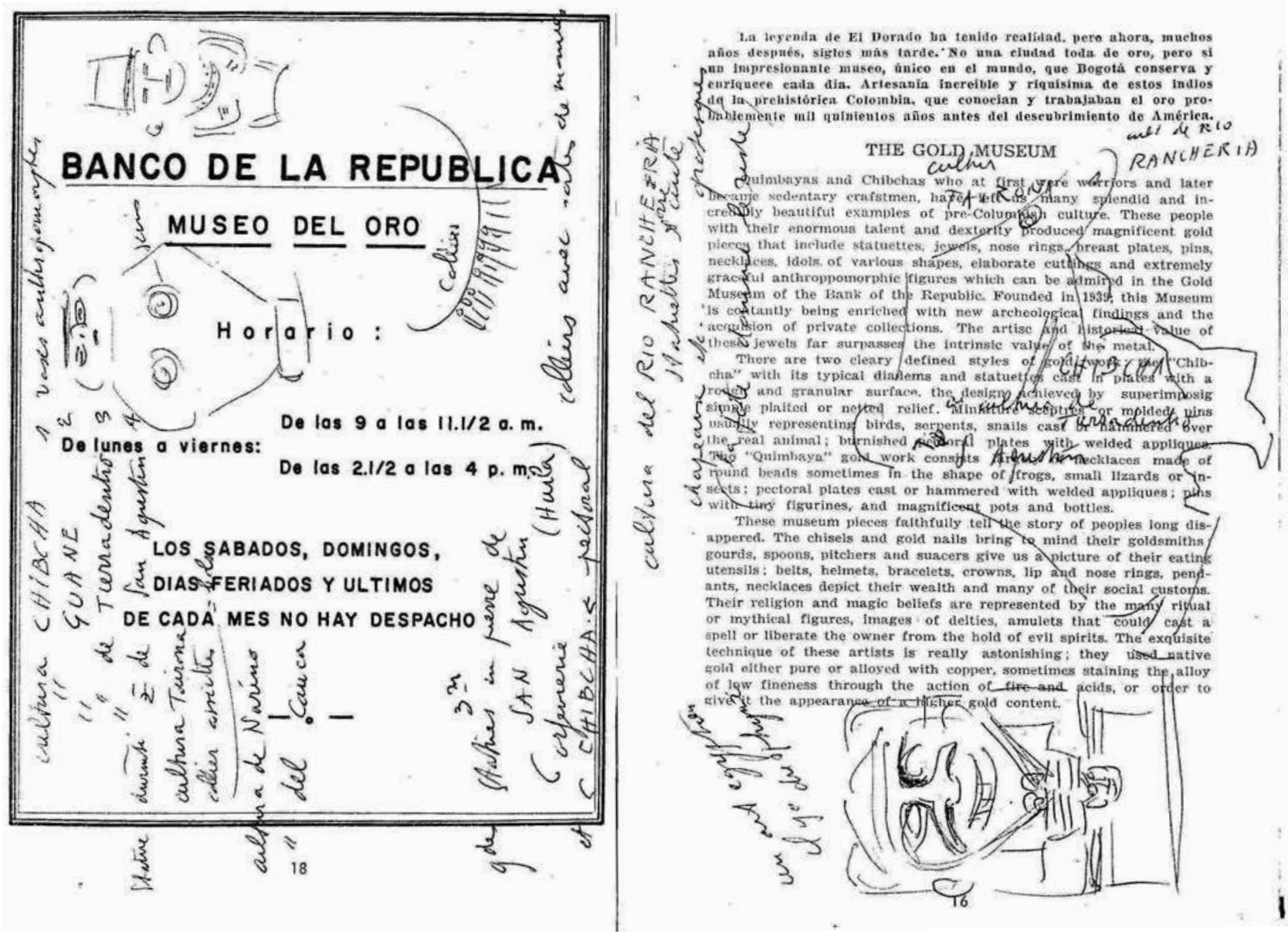

Fig. 4 Documents du Museo del oro, Bogota, annotés par Yves Laloy. Collection Pascal Laloy.

microcosme ont été à la base de ses créations plastiques. C'est par un double vocabulaire, géométrique et figuratif, qu'il les a représentés, ce caractère polymorphe étant l'une des caractéristiques de son talent. Cependant, ainsi que nous l'avons vu, c'est un souffle surréaliste qui emplit l'œuvre de cet artiste inspiré, même si ce dernier s'en défendait. Il affirmait ainsi dans un entretien avec un journaliste péruvien qu'il considérait « cette tendance picturale [le surréalisme] comme une erreur, qu'elle était négative et même nuisible ». Très intéressé par l'art hispanique et préhispanique, Laloy s'était beaucoup documenté sur les civilisations anciennes, leur art, leurs rites, leurs mythes et leurs religions. Dire qu'il n'a pas eu de modèle n'est pas tout à fait juste. Il avait une façon d'être, de penser et de peindre en accord avec le surréalisme et a participé à quatre expositions surréalistes collectives. Les musées ne s'y sont d'ailleurs pas trompés, en exposant les œuvres de Laloy, y compris les compositions géométriques, dans les salles consacrées à ce mouvement. Vis-à-vis des arts non occidentaux, il a intégré plus ou moins consciemment les formes dont il avait eu connaissance, d'abord par les livres avant de les voir in situ. Il les a transformées, modelées, et leur assimilation plastique participe assurément de l'effet de sidération que l'œuvre de Laloy induit sur le spectateur.

mots clés / keywords : primitivisme // primitivism • abstraction // abstraction $\bullet$ surréalisme // surrealism $•$ spiritualité // spirituality • magie // magic.

Université Paris IV-Sorbonne sjduco.catalogue-laloy@wanadoo.fr 
1920

Yves Laloy naît le 13 juin à Rennes. Issu d'une famille d'architectes, il étudie à l'École nationale des beaux-arts de Paris dans la section Architecture.

1946

Il épouse Jeanne Beauregard.

\section{7}

Muni de son diplôme d'architecte, il exerce sa profession à Rennes dans le cabinet de son père et commence à peindre quelques paysages conventionnels qu'il expose à la galerie d'Orsel à Paris.

1952

Il abandonne l'architecture pour se consacrer définitivement à la peinture. Son art évolue vers des constructions géométriques rigoureuses ou biomorphiques.

\section{5}

Peu avant le conflit de Suez, il part à bicyclette pour faire le tour du Bassin méditerranéen. Après des étapes au Maroc et en Libye, il arrive au Caire. Pris pour un espion, il est emprisonné. Après sa libération, il a le temps de voir Louksor.

\section{8}

Par l'intermédiaire de Géo Dupin, responsable de la galerie L'Étoile scellée, il fait la connaissance d'André Breton. Celui-ci organise le 7 octobre une exposition d'une trentaine de tableaux d'Yves Laloy dans la galerie La Cour d'Ingres à Paris. En dépit de ses relations avec Breton, Laloy, artiste réservé, indépendant, n'appartiendra jamais au mouvement surréaliste ni à aucun autre groupe.

\section{0-1962}

Voyages en Espagne, au Canada, ainsi qu'à New York à l'occasion de l'exposition surréaliste $E R O S$, dans laquelle il est représenté. Ayant un besoin constant d'évasion, il participe à deux campagnes de pêche de six mois jusqu'à Terre-Neuve, partageant les conditions de vie rudes des marins.

\section{5}

Yves Laloy est représenté dans l'exposition L'Écart absolu à Paris.

Grâce à une cliente péruvienne de $M^{\text {me }}$ Laloy, qui tenait une galerie à Paris, il expose une vingtaine de tableaux dans une galerie de Lima, l'Art Center. Il reste un mois à Lima. La première des œuvres exposées est un hommage au pays qui l'accueille: Je suis breton, je suis la loi, vive la France, viva el Perú. Il visite plusieurs musées de la ville, se rend à Cuzco et au Machu Picchu. Il se rend ensuite en Colombie et visite le Museo del Oro de Bogotá, d'où il rapporte quelques documents. Il s'est peu exprimé sur son voyage au Pérou mais a enfoui dans sa mémoire la plupart de ses souvenirs.

\section{0-1971}

Rédaction d'un livre, non édité, La Beauté et ses à-côtés.

\section{2-1973}

Plusieurs expositions, à Paris, Milan (galerie A. Schwarz et Galleria del Levante), Bâle (galerie Schreiner), présentent des œuvres d’Yves Laloy.

1989

Il participe à des expositions individuelles et collectives à Bâle et à Milan.

1996

Le galeriste Carl Laszlo présente plusieurs œuvres d'Yves Laloy dans une exposition collective à Budapest.

\section{9}

Yves Laloy meurt à Cancale le 8 septembre. Une rétrospective d'une centaine de ses œuvres a été organisée en 2004 par le musée des Beaux-Arts de Rennes, sa ville natale.

\section{Annexe 2 : musées dont les collections permanentes comprennent des œuvres d'Yves Laloy}

\section{En France}

Musée national d'Art moderne à Paris : Soleil sur l'Ô (don des héritiers de l'artiste en 2005).

Musée de Grenoble : deux tableaux (don de Miklos von Bartha).

Musée des Beaux-Arts de Rennes, quatre tableaux, dont: Les petits pois sont verts... les petits poissons rouges (ancienne collection André Breton, préemption vente hôtel Drouot 2003).

\section{À l'étranger}

Galleria Nazionale d'Arte Moderna e Contemporanea à Rome : quatre tableaux (don d'Arturo Schwarz).

Israel Museum à Jérusalem : deux tableaux (don d'Arturo Schwarz). 


\section{Bibliographie}

\section{Ouvrages généraux}

\section{AnATI, Emmanuel}

2005 L'Art du tapa. Étoffe pour les Dieux, étoffe pour les Hommes. Paris, L'Insolite.

\section{BAHTI, Mark}

2003 A Guide to Navajo Sand Paintings. Tucson, Rio Nuevo Publishers.

\section{BAUdelaire, Charles \\ 1980 [1857] « Critique d'art », in Euvres complètes. Paris, Robert Laffont \\ [«Bouquins 》]. \\ BENOIST, LUC \\ 2004 [1975] Signes, symboles et mythes. Paris, PUF [«Que sais-je ? »]. \\ Breton, André \\ 1965 Le Surréalisme et la Peinture. Paris, Gallimard. \\ 1968 [1947] Signe ascendant. Paris, Gallimard [«Poésie 》]. \\ Breton, André et Legrand, Gérard 1991 [1957] L'Art magique. Paris, Phébus. \\ FeEst, Christian \\ 1994 Les Indiens d'Amérique du Nord. Paris, Thames and Hudson. \\ FREUD, Sigmund 1965 [1912] Totem et tabou. Paris, Payot.}

\section{Maguet, Frédéric}

2006 « Des Indiens de papier, entre réception royale et réception populaire », Gradhiva 3 , nouvelle série : 89-102.

\section{Pierre, José}

1967 « Les petits pois sont verts... les petits poissons rouges 》, carton d'invitation de l'exposition Yves Laloy. Milan, Galleria del Levante.

\section{PRINZHORN, Hans}

1984 [1922] Expressions de la folie, dessins, peintures et sculptures d'asile. Paris, Gallimard

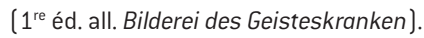

\section{RANK, Otto}

1998 [1932] L'Art et l'artiste. Paris, Payot [«Bibliothèque scientifique »].

\section{Catalogues d'exposition}

1941 Indian Art in the United States, Frederic H. Douglas et René d'Harnoncourt. New York, Museum of Modern Art.

1958 Laloy présenté par André Breton. Paris, galerie La Cour d'Ingres.

1984 RuBIN, William, Primitivism in the 20th century art: affinity of the tribal and the modern. New York, Museum of Modern Art (éd. française : Paris, Flammarion, 1987).
1967 Yves Laloy. Milan, Galleria del Levante. 1986 Tuchman, Maurice, The Spiritual in Art, Abstract painting 1890-1985. Los Angeles, County Museum of Art.

2003 André Breton, 42 rue Fontaine, vente aux enchères de la collection André Breton. Paris, salle Drouot-Richelieu.

2003 Tableaux kuna. Les molas, un art d'Amérique. Paris, bibliothèque Forney- Arthaud. 2004 Yves Laloy. Rennes, musée des BeauxArts-Apogée.

2006-2007 D'un regard l'autre. Histoire des regards européens sur l'Afrique, l'Amérique et l'Océanie. Paris, musée du Quai Branly-Réunion des musées nationaux.

Expositions surréalistes auxquelles Yves Laloy a participé

1959-1960 Exposition internationale du Surréalisme : $\varepsilon R O S$. Paris, galerie Daniel Cordier. 1960 Surrealist intrusion in the Enchanter's domain. New York, D'Arcy Galleries.

1961 Mostra internazionale del surrealismo. Milan, galerie Schwarz.

1965 Exposition internationale du surréalisme. L'Écart absolu. Paris, galerie de l'CEil.

\section{Résumé / Abstract}

Suzanne Duco, Yves Laloy. Un dialogue entre primitivisme et surréalisme. - Architecte de formation, Yves Laloy commence à peindre vers 1950. André Breton a découvert son œuvre avec admiration en 1958 et la compare aux tableaux de sable des Indiens navajo. L'art de Laloy est marqué par un double vocabulaire : des compositions géométriques d'une grande audace picturale et des tableaux figuratifs empruntant des thèmes au surréalisme, bien que l'artiste ait récusé ce mouvement. Les compositions géométriques, qui sont des itinéraires spirituels, peuvent être mises en regard avec l'art tribal. Quant aux personnages, grotesques ou tragiques, ils ont des traits communs avec les masques terrifiants des Amériques. II n'y a pas d'influence directe, mais une identité de la démarche spirituelle. Ainsi, le dialogue fécond entre le surréalisme et les arts non occidentaux est présent dans une grande partie de l'œuvre de Laloy.
Suzanne Duco. Yves Laloy. Primitivism and surrealism: a dialogue between two cultures. - After training as an architect, Yves Laloy began to paint around 1950. André Breton discovered his work around 1958, admired it and compared it with Navajo Indian sand paintings. Yves Laloy's art is characterised by a double vocabulary: geometrical compositions of great pictorial audacity and figurative paintings borrowing themes from surrealism, although the artist had objected to the surrealist movement. The geometrical compositions, which are spiritual itineraries, can be compared with tribal art. The characters tend to be grotesque or tragic, and have common features with the terrifying masks of the Americas. There is no direct influence, but a common spiritual identity. Thus, the fertile dialogue between surrealism and non-Western arts is present in much of the work of Yves Laloy. 